\title{
Avoiding Unnecessary Cost in Nursing Homes: An Achievable Task
}

\author{
Firas Kawtharani* \\ Orthopedic Surgery, Sports Medicine and Trauma, USA \\ *Corresponding author: Firas Kawtharani, Orthopedic Surgery, Sports Medicine and Trauma, Southwest Medical Center, Liberal, Kansas-67901, USA
}

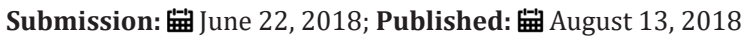

\begin{abstract}
In orthopedic surgery and other surgical specialties, reliance on long term facility care is increasing and this fact is partly explained by the increase in life expectancy as well as the recent implementation of bundled payment reimbursement. Nursing homes are currently pivotal in the care of the orthopedic patient and the quality of care these facilities provide help in preventing possible complications that might affect surgical patients. The main challenge presented to these institutions is that of the high hospitalization rate of nursing home residents [1-4]. The article addresses the problem through a review that defines it further and elaborates on its potential ramifications. The latest evidence is presented with regards to providing practical solutions that will save significant resources and make the healthcare system as efficient as possible in delivering the best and most comprehensive care to our communities.
\end{abstract}

\section{Introduction}

The nursing home population is extremely frail and vulnerable and requires delicate care [5]. Nevertheless, there is much evidence that a lot of these hospitalizations may be preventable [1-4]. This setback causes unnecessary healthcare expenditure amounting to 47 million dollars in 2006 solely, in the state of Georgia [3]. In addition, unnecessary hospital admissions cause discomfort and anxiety to residents and their families, iatrogenic and nosocomial conditions and waste of resources that could be potentially used to treat critically ill patients [4].

From a public health standpoint, there is a lack of a nationwide standard approach in treating nursing home residents, which is draining the healthcare system. In fact in recent years there was a surge in the creation of new long-term care facilities and home health agencies due to the increase in life expectancy of the U.S population. However the number of geriatric specialists and skilled primary care nurses did not increase enough to meet the demands of these organizations [3].

In addition, there is a lack of organizational infrastructure, namely practice guidelines, cares paths, communication, leadership and proper implementation of advanced directives in nursing homes [3]. The frequent absence of primary care specialists mainly advanced practice registered nurses (APRN) compromises the ability of nursing homes to properly triage ill patients that need hospitalization. It prevents as well delivering adequate treatment that will avoid a hospital visit in more than half of the incidents [1].

The appropriate solution is to formulate a national strategy to counter the unnecessary spending and channel these wasted resources to treat patients who are truly in need for hospitalization. This process started with an initiative launched by the center for Medicare and Medicaid services: CMS that collaborated with major academic centers in providing appropriate resources to nursing homes [2] for those to minimize their rate of hospitalization. Ultimately the model adopted will pay reimbursements according to the performance of each facility in a value-based approach. The initiative is championed by APRN's who are involved in direct treatment, education, leadership and the enhancement of communication in a minimally punitive environment. This effort is ideal and proved in multiple pilot studies to drop down the rate of hospitalization to more than $30 \%$ as in the case of Missouri [1]. APRN's provide expertise in assessing acute changes in condition; better access to services such as laboratory, X-ray, and intravenous fluid administration as well as proper implementation of advanced directives. In addition, they act as a liaison between nursing home workers and a visiting geriatric specialist who prove resourceful in challenging situations. Progress in minimizing hospitalization can be followed up with periodic quality assessments to evaluate the results and implement policies and plans that will improve further nursing home interventions such as administering medications, IV fluid resuscitation, laboratory evaluations, preventing fall episodes, metabolic and gastro-intestinal disturbances etc... To elaborate further on the cost benefit analysis with regards to this initiative, we use again the example of Georgia in 2006 in which the avoidable health care cost due to unnecessary hospitalization was around 47 million dollars. This amount may support the availability of an APRN 5 days per week in every nursing home at an average cost of $\$ 100,000$ in salary and benefits and have savings remaining [3]. 
The hiring of an APRN in a specific nursing home can be done immediately however reaping the benefits of organizational change would take at least a year. Multiple pieces of the puzzle should be put together to construct a solid system with proper leadership and hierarchy. For instance in Missouri, the change in policy initiative showed tangible change from 2014 to 2015 (The initiative was associated with 5.9 percentage-point decrease in the probability of having any hospitalization in 2014 and a 9.3-percentage-point decrease in 2015) [2]. Nursing home administrators are required to provide necessary resources for the nursing leaders and the APRN's who are responsible in ensuring proper algorithms in dealing with emerging medical situations among residents. Their skilled approach is needed to avoid unnecessary hospitalizations. The rest of the nursing home personnel are the workforce in the frontlines who deliver appropriate care for the residents ideally in a constructive non-judgmental environment. Their primary assessment and communication is pivotal in delivering appropriate care. Additional policy changes should implement quality improvement programs such as Interventions to Reduce Acute Care Transfers (INTERACT) [2], attempt to minimize medication errors, fall risks and improve communication among nursing home staff. The hope is to effectively diagnose emerging conditions among residents such as pneumonia, GI and metabolic disturbances etc... and orchestrate with the administration a proper workup including timely lab results, imaging and treatment measures such as IV fluid administration and if need be antibiotics. As mentioned previously, it is necessary to evaluate progress through serial interdisciplinary meetings that discuss errors, improvement and plan ahead using concrete data.

\section{Conclusion}

In conclusion, change of culture in nursing homes is necessary to promote an environment of safety [5]. Providing nursing homes with proper resources whether APRN or qualified nursing leaders who oversee a smooth flow of events is paramount. Personnel should be encouraged to report errors in drug administration, falls, and other adverse events in a minimally punitive environment. State of the art technology in assessing patients should be implemented such as use of updated EMR's. All of these measures are being proved through pilot studies to decrease dramatically unnecessary hospitalization [1-4]. They help as well in saving millions of tax payers' dollars that could be used to treat and attend to patients who truly need it.

\section{References}

1. Rantz MJ, Popejoy L, Vogelsmeier A, Galambos C, Alexander G, et al. (2017) Successfully reducing hospitalizations of nursing home residents: results of the missouri quality initiative. J Am Med Dir Assoc 18(11): 960-966.

2. Ingber MJ, Feng Z, Khatutsky G, Wang JM, Bercaw LE, et al. (2017) Initiative to reduce avoidable hospitalizations among nursing facility residents shows promising results health affairs. Health Aff (Millwood) 36(3): 441-450.

3. Ouslander JG, Lamb G, Perloe M, Givens JH, Kluge L, et al. (2010) Potentially avoidable hospitalizations of nursing home residents: frequency, causes, and costs. J Am Geriatr Soc 58(4): 627-635.

4. Ouslander JG, Perloe M, Givens JH, Kluge L, Rutland T, et al. (2009) Reducing potentially avoidable hospitalizations of nursing home residents: results of a pilot quality improvement project. J Am Med Dir Assoc 10(9): 644-652.

5. Cawiezell SJ, Vogelsmeier A, Mckenney C, Rantz M, Hicks L, et al. (2006) Moving from a culture of blame to a culture of safety in the nursing home setting. Nursing Forum 41(3): 133-140.
Creative Commons Attribution 4.0 International License

For possible submissions Click Here

\section{Submit Article}

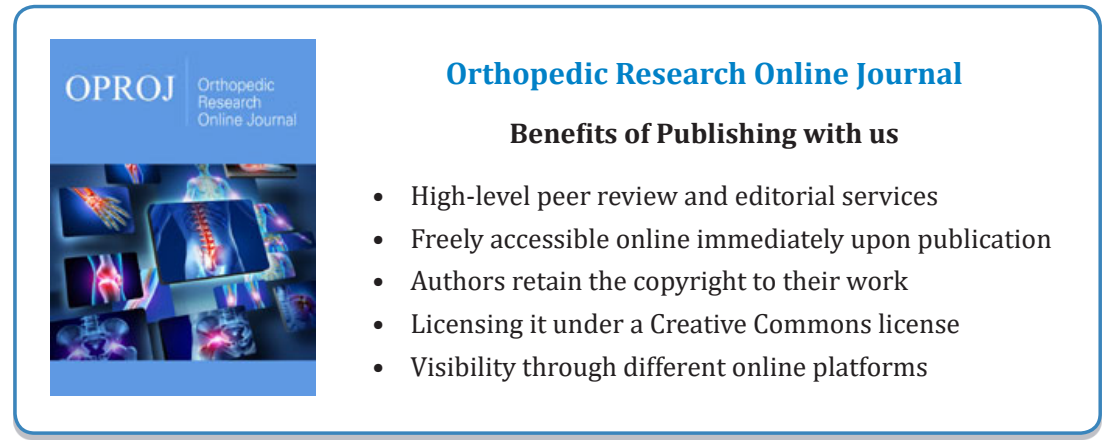

